
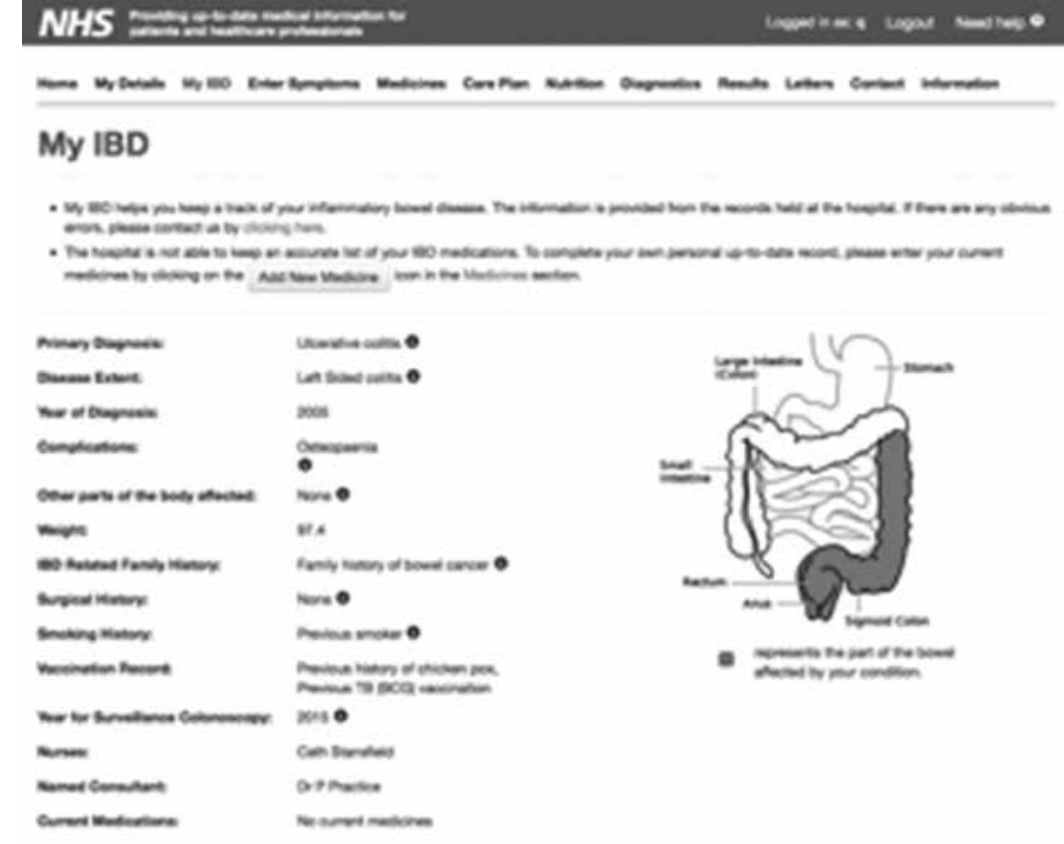

Abstract 0C-082 Figure

\section{Colorectal free papers}

\section{OC-083 FAECAL OCCULT BLOOD TEST ANALYSIS IN THE UNITED KINGDOM BOWEL CANCER SCREENING PROGRAMME}

doi:10.1136/gutjnl-2013-304907.082

1."J Geraghty, ${ }^{2} \mathrm{~J}$ Snowball, ${ }^{2} \mathrm{P}$ Butler, ${ }^{1} \mathrm{~S}$ Sarkar, ${ }^{3 R}$ Blanks, ${ }^{2} \mathrm{~S}$ Halloran, ${ }^{4} \mathrm{C}$ Rees. ${ }^{1}$ Royal Liverpool University Hospital, Liverpool; ${ }^{2}$ Southern Bowel Screening Hub, Guilford; ${ }^{3}$ Cancer Epidemiology Unit, University of Oxford, Oxford; ${ }^{4}$ South Tyneside NHS Foundation Trust, South Shields, UK

Introduction Increased vascularity of colorectal neoplasia creates bleeding detected by Faecal Occult Blood tests (FOBt). As bleeding is sporadic \& unevenly distributed within stools, multiple testing may be required. UK bowel cancer screening programme (BCSP) kits contain 6 windows \& subjects returning 5 or 6 positive results are termed "Abnormal" \& referred to colonoscopy. If 1-4 windows are positive, the result is initially "Unclear" \& 2 further kits are submitted, further positivity leads to colonoscopy ("Weak positive"). If no further blood is detected, subjects are deemed "Normal" \& retested in 2 years.

Aim to study FOBt positivity in detail \& whether particular patterns are associated with neoplasia rates that indicate the screening algorithm should be changed.

Methods We selected all subjects from one hub completing 2 screening episodes between 2007-9. Each episode included up to 3 kits and 18 windows. 95 possible combinations were identified. The number of positive windows compared to the total in a given episode was expressed as a "positivity ratio", ranging from $0-100 \%$. Each combination leading to colonoscopy was analysed. Abnormal (83-100\% positivity) \& Unclear (11-83\% positivity) groups were matched to neoplasia detection rates. Subjects with cancer detected in episode 2 following an Unclear result in episode 1 , had their episode 1 pattern analysed.

Results FOBt from 284,387 subjects resulted in 4,000 colonoscopies, diagnosing 286 cancers. The overall cancer rate was $7.1 \%$ \& adenoma rate $39.9 \%$. The cancer rate was $21.3 \%$ in the Abnormal group and $5.8 \%$ in the Weak positive group. Cancer detection increased from $1.9-24.5 \%$ in linear correlation with increasing positivity of windows, ranging from $11-83 \%$ of windows positive. Equivalent percentage positivity rates may or may not lead to colonoscopy depending on the particular pattern. A combination of 4 positive windows in kit 1 followed by 2 normal kits ( $4 \mathrm{NN}$ ) equates to a positivity rate of $22 \%$ \& is currently categorised in the Normal group \& doesn't lead to colonoscopy. Other combinations with $22 \%$ window positivity do lead to colonoscopy $\&$ a cancer detection rate of $3 \%$. There were 260 subjects with a $4 \mathrm{NN}$ combination in episode 1 not leading to colonoscopy \& 5 of these subsequently had cancers detected following different combinations in episode 2 .

Conclusion This study demonstrates higher ratios of positive windows; detect higher rates of cancer. At present, in the UK some subjects with $11 \%$ positive windows proceed to colonoscopy, while others with a rate of $22 \%$ (all at kit 1 ) do not. Based on these findings, further work examining the entire BCSP population, including the costs \& benefits of changing the algorithm is in progress.

Disclosure of Interest None Declared

\section{OC-084 A COMPARATIVE STUDY OF CLINICAL OUTCOMES AFTER LAPAROSCOPIC OR OPEN RESTORATIVE PROCTOCOLECTOMY}

doi:10.1136/gutjnl-2013-304907.083

1,*I White, ' IJ T Jenkins, 'R Coomber, 'S K Clark, 'R K S Phillips, 'R H Kennedy. 'Department of Surgery, St Mark's Hospital, London, UK

Introduction Laparoscopic colorectal surgery confers significant benefits over similar open procedures. A paucity of data exists examining this in restorative proctocolectomy, although recent literature has suggested reduced adhesions and improved fertility. We assessed consecutive patients undergoing laparoscopic ileal pouch anal anastomosis (IPAA), comparing clinical outcomes with those following conventional open surgery.

Methods 207 consecutive patients undergoing IPAA between November 2006 and November 2011 were assessed [76 (37\%) laparoscopic procedures and $131(67 \%)$ open cases were included]. 
A careful collaborative approach to developing the new technique was undertaken during the first two years in this high volume centre. Data were collected prospectively on a dedicated database. Study end points included post-operative length of stay, 30 day morbidity [Clavien-Dindo classification(C-D)], readmission, reoperation, pouch function \& failure.

Results There were no significant differences in patient age, sex, BMI or previous abdominal surgery between the two groups. Conversion rate was 9\%; Median operative time was significantly shorter for open surgery - 208 (IOR 178-255) versus 285 minutes (IOR 255-325); $p<0.0005$. The duration of laparoscopic surgery decreased significantly during the study period.

Laparoscopy significantly reduced length of stay: median 6 days (IOR 4.25-8), v 8 days (IOR 7-12); $\mathrm{p}<0.0005$.

Minor [C-D I/II] complications were significantly reduced with laparoscopy (32.8\% v 50.4\%: OR 0.48 [95\%CI 0.27-0.87]). Complications [all grades] were reduced non-significantly after laparoscopic surgery There were no significant differences in total complications $-51.3 \%$ after laparoscopy versus $61.5 \%$ : OR 0.66 [95\%CI 0.37-1.17], anastomotic leak rate, major morbidity, 30 day readmission, reoperation and stoma closure rates.

Pouch failure has occurred in 14 patients (7.7\%) overall, however there were $12(11 \%)$ in the open group with only $2(2.6 \%)$ in the laparoscopic group, although this is not statistically significant $(\mathrm{P}=0.172)$. No significant difference was seen in pouch dysfunction rates.

Conclusion Laparoscopic restorative proctocolectomy significantly reduces length of stay and minor morbidity and can be offered to an increasing proportion of restorative proctocolectomy patients. A careful collaborative developmental process has occurred in a high volume centre to achieve these results.

Disclosure of Interest I. White: None Declared, J. Jenkins: None Declared, R. Coomber: None Declared, S. Clark: None Declared, R. Phillips: None Declared, R. Kennedy Grant/Research Support from: Ethicon Endosurgery and Olympus UK

\section{REFERENCE}

Dindo D., Demartines N., Clavien P.A.; Classification of Surgical Complications. Ann Surg. 2004; 244: 931-937.

\section{OC-085 PREDICTING LOCAL OR SYSTEMIC RECURRENCE AFTER CURATIVE RESECTION FOR COLORECTAL CANCER: THE ROLE OF ELASTICA STAINS TO OPTIMISE DETECTION OF VENOUS INVASION}

doi:10.1136/gutjnl-2013-304907.084

1."E Wright, ' $\mathrm{C}$ C McAteer, 'D C McMillan, 'P G Horgan, ${ }^{2} \mathrm{~A}$ K Foulis, 'C S Roxburgh. 'Academic Unit of Surgery, University of Glasgow; ${ }^{2}$ University Department of Pathology, NHS Greater Glasgow and Clyde, Glasgow, UK

Introduction Following colorectal cancer resection, high-risk tumour pathology guides provision of adjuvant therapy and follow-up. Currently, routinely assessed features include local invasion, nodal status, venous invasion (VI), serosal invasion, differentiation and margin status. It was recently reported that elastica tissue stains increase frequency of detection of VI to $>50 \%$, reducing interobserver variation, increasing its prognostic value (Roxburgh 2010, Kirsch 2013). Given recurrence is either local, systemic or both, we sought to examine the role of routinely assessed pathological criteria including VI (detected using elastica) in determining recurrence following resection for colorectal cancer.

Methods From an institutional database 555 patients undergoing curative resection between 1997-2009 were identified with recurrence and follow-up data available. Pathology data was taken from reports issued at the time. VI was assessed prospectively with routine elastica staining for 417 patients and retrospectively in 138. Analysis was performed with binary logistic regression. Due to a high number of comparisons, to enter the multivariate model a significance level of $<0.01$ was used.

Results Of 555 patients, 141 (25\%) developed recurrence. Frequency of VI detection was $54 \%$. On logistic regression, elastica detected VI, T stage, lymph node involvement, serosal involvement, margin involvement, tumour perforation, peri-tumoural inflammation (Klintrup grade) and tumour necrosis were predictors of recurrence (any site, all $\mathrm{P}<0.05$ ). Differentiation was not. On multivariate analysis VI (OR $3.27, \mathrm{P}<0.001)$, lymph node involvement (OR 2.34, $\mathrm{P}=0.005$ ), serosal invasion ( $\mathrm{OR} 2.38, \mathrm{P}=0.005)$, Klintrup grade (OR $0.68, \mathrm{P}=0.037$ ) were independently predictors of recurrence. Most recurrence was systemic $(75 \%)$. The same features predicted systemic recurrence as did overall recurrence but on multivariate analysis, only VI (OR 2.90, P = 0.004), lymph node involvement ( $\mathrm{OR} 2.27, \mathrm{P}=0.012$ ) and necrosis ( $\mathrm{OR} 1.63, \mathrm{P}=0.013$ ) were independent predictors of systemic recurrence. In the 35 cases of local recurrence VI, T stage, lymph node involvement, serosal involvement and margin involvement were significantly related $(\mathrm{P}<0.05)$. On multivariate analysis, only VI (OR $2.28, \mathrm{P}=0.057)$ and $\mathrm{T}$ stage (OR 2.53, $\mathrm{P}=0.003$ ) were independent predictors.

Conclusion Whilst several pathological features predict local and systemic recurrence after surgery, VI detected at increased frequency (54\%) with elastica stains was the only consistent, independent predictor of recurrence, at least as important as nodal spread. These results support implementation of routine measures such as elastica staining to optimise reporting of VI.

Disclosure of Interest None Declared

\section{OC-086 SURVIVAL BENEFIT OF FDG-PET ORIENTED SURGERY FOR RECURRENT COLORECTAL CANCER}

doi:10.1136/gutjnl-2013-304907.085

"H Ishikawa, 'A Watanabe, 'T Mukogawa, 'M Nakatani, 'Y Nishiguchi, 'M Matsusaka, 'S Ko. 'Surgery, Nara Prefectural Nara Hospital, Nara city, Japan

Introduction Pivotal treatment for localised recurrent colorectal cancer is surgical resection. Fluorine-18 fluorodeoxyglucose positron emission tomography (FDG-PET) has been accepted as an effective tool to identify disease localizations for patients with known or suspected recurrent colorectal cancer.

This study is to analyse the survival benefit of FDG-PET on the diagnosis and indication of surgical intervention for

Methods Consecutive 61 patients, with known or suspected recurrence of colorectal cancer based on elevation of tumour markers or abnormal findings on the follow-up CT image, underwent FDG-PET for 85 times between December 2003 and September 2009. Patients were aged between 39 and 94 years (median 66); 35 were male, 22 were Duke's A or B stage, and 31 had a history of colon cancer. The average period between operation and first FDGPET was 24 months (range 4-114). Of 61 cases 50 had elevated serum CEA or CA19-9 (82.0\%). For each case the diagnosis of FDGPET image was compared with that of CT image and the final diagnosis.

Results Recurrence developed 2 times in average (range 1-6).

Of 61 patients five were identified recurrence by FDG-PET solely and indicated operation for 7 times. One of four shows disease-free survival for 70 months after common iliac replacement operation.

FDG-PET showed one false - positive and three false - negative findings. Totally, the sensitivity of FDG-PET was $93.3 \%$ and its accuracy was $91.8 \%$, whereas those of CT were $85.0 \%$ and $83.6 \%$, respectively.

Of 61 patients 19 had diffused disease spread by FDG-PET findings and were judged as contraindication of operation and the other 42 with localised disease spread received operation for 90 times (liver 28, lung 14, local 37, local with distant meta 5, etc.). Cumulative 1 -year and 3 -year survival ratios of operation cases after first recurrence were $95 \%$ and $80 \%$, whereas, those of contraindication 